\title{
Tracing part-per-billion line shifts with direct-frequency-comb Vernier spectroscopy
}

\author{
M. Siciliani de Cumis, ${ }^{1}$ R. Eramo, ${ }^{1}$ N. Coluccelli, ${ }^{2}$ M. Cassinerio, ${ }^{2}$ G. Galzerano, ${ }^{2}$ P. Laporta, ${ }^{2}$ \\ P. De Natale, ${ }^{1}$ and P. Cancio Pastor ${ }^{1, *}$ \\ ${ }^{1}$ Istituto Nazionale di Ottica-CNR and European Laboratory for Non-linear Spectroscopy, Via N. Carrara 1, Sesto Fiorentino, Italy \\ ${ }^{2}$ Dipartimento di Fisica-Politecnico di Milano and Istituto di Fotonica e Nanotecnologie-CNR, Piazza Leonardo da Vinci 32, Milano, Italy
} (Received 26 June 2014; published 14 January 2015)

\begin{abstract}
Accurate frequency measurements of molecular transitions around $2 \mu \mathrm{m}$ are performed by using a direct-frequency-comb spectroscopy approach that combines an $\mathrm{Er}^{+}$frequency-comb oscillator at $1.5 \mu \mathrm{m}$, a Tm-Ho fiber amplifier, and a Fabry-Perot-filter, high-resolution dispersive spectrometer optical multiplex-detection system. This apparatus has unique performances in terms of a wide dynamic range to integrate the intensity per comb mode, which allows one to measure molecular absorption profiles with high precision. Spectroscopic information about transition frequencies and linewidths is very accurately determined. Relative frequency uncertainties of the order of a few parts in $10^{-9}$ are achieved for rovibrational transitions of the $\mathrm{CO}_{2}$ molecule around $5100 \mathrm{~cm}^{-1}$. Moreover, tiny frequency shifts due to molecular collisions and interacting laser power using direct comb spectroscopy are investigated in a systematic way.
\end{abstract}

\section{INTRODUCTION}

Unprecedented results in molecular spectroscopy, in terms of resolution, precision, and sensitivity over a broad spectral range, have been widely demonstrated using frequency-comb lasers (FCLs) [1] as light sources in the framework of direct comb spectroscopy (DCS). Actually, demonstrations in almost all electromagnetic spectral regions, from UV [2], through visible-NIR [3-7] and mid-IR [8-10], up to the THz range [11], may open new opportunities in physics, chemistry, biology, and industry. Indeed, DCS-based instrumentation developed for multiple trace gas sensing [9,12], hyperspectral lidar [13], scanning near-field optical microscopy (SNOM) [14], and/or coherent Raman spectroimaging [15] are just a few examples of DCS applicability, spanning from environmental and climatic change monitoring to single-virus vibrational mapping as well as chemical recognition of nanomaterials.

Nowadays, DCS demonstrations are mainly pushing on sensitive detection of highly multiplexed spectra of molecules by increasing speed, resolution, spectral bandwidth [16-19], or optical absorption path length $[3,4,7,20]$, with frequency calibration provided by known spectral lines. However, only a few of them [21,22] make use of the precision and the accuracy of the underlying FCL for frequency measurements. Improvement of the spectroscopic frequency data of the targeted molecules at $10^{-9}$ uncertainty level, from such coherent-dual-comb DCS experiments, could have a direct impact on frequency calibration applications, from fiber-optics communications to broadband astronomical spectrographs. However, this technique is rather complex and cumbersome, requiring two optical frequency combs, and systematic effects due to the parallel interaction of multiple optical frequencies are difficult to calibrate in an accurate way. Here, we present a DCS approach that combines a Fabry-Perot (FP)-filtered FCL with a diffraction limited dispersive spectrometer to perform comb-tooth-resolved spectroscopy, providing molecular spectra against an absolute optical frequency scale. It should be

*pablo.canciopastor@ino.it; www.ino.it, www.lens.unifi.it. noted that this approach has a close similarity to the use of a set of single frequency lasers, relying on a highly dispersive system. Therefore, it is able to measure with high fidelity the variable intensity dynamics along the spectral line shapes, thus shedding light on tiny systematic effects affecting frequency measurements or critical line profile determinations [23,24]. In this respect, our setup can provide a higher accuracy, as compared to dual-comb spectroscopy [21,22], though at the price of a longer acquisition time when investigating broad spectral regions. Fundamental physics experiments, such as the investigation of possible time-reversal symmetry violation [25] or time variation of fundamental constants [26] performed with ultracold molecules, could also benefit from the broad spectral coverage, frequency, and intensity determination accuracy of our DCS.

\section{EXPERIMENT}

Our DCS spectrometer, shown schematically in Fig. 1, combines a NIR-FCL amplified in the $2 \mu \mathrm{m}$ region by using a homemade Tm-Ho-doped fiber amplifier (FA), a medium finesse FP cavity, a diffraction limited grating dispersive spectrometer (SOPRA) [27], and an IR CCD camera for multiplex detection of FP transmitted comb modes. It follows the comb-Vernier dispersive approach [4] with a Vernier ratio of about 18/1. In this way, the Vernier FP transmits a comb pattern spaced by the FP free spectral range (FSR) that can be resolved by the $1.5 \mathrm{GHz}$ resolution of SOPRA.

The NIR-FCL is based on a $\mathrm{Er}^{+}$-doped fs mode-locked laser, emitting at around $1.5 \mu \mathrm{m}$, with a repetition rate $f_{r}=$ $250 \mathrm{MHz}$. It is a self-referenced comb system (MENLO FC1500), with both $f_{r}$ and carrier-envelope-offset frequency ( $f_{\mathrm{CEO}}$ ) that are phase locked against a $\mathrm{Rb} /$ global positioning system (Rb/GPS)-disciplined $10 \mathrm{MHz}$ quartz oscillator, with a stability of $6 \times 10^{-13}$ in $1 \mathrm{~s}$ and an absolute frequency accuracy of $2 \times 10^{-12}$.

A fraction of the NIR-FCL, about $10 \mathrm{~mW}$, at $1.55 \mu \mathrm{m}$ is amplified by a homemade $\mathrm{Er}^{+}$-doped FA up to $0.5 \mathrm{~W}$, maintaining a transform-limited pulse duration of $50 \mathrm{fs}$. A highly nonlinear fiber broadens the amplified light to an 


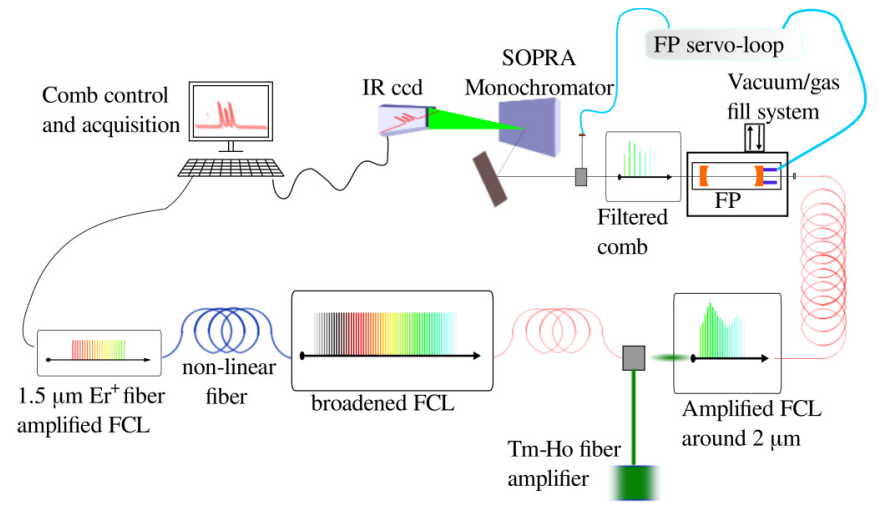

FIG. 1. (Color online) Experimental setup for DCS around $2 \mu \mathrm{m}$.

octave-spanning supercontinuum (SC), from 1 to $2.1 \mu \mathrm{m}$. Since the power per mode of the SC is too low $(<100 \mathrm{nW})$ for comb-tooth-resolved DCS, we implemented a Tm-Ho FA [28] to boost the power of the SC long-wavelength tail, around $2 \mu \mathrm{m}$, up to $30 \mu \mathrm{W} /$ tooth, corresponding to a total average power of $1.5 \mathrm{~W}$, quite sufficient for our purposes. After coupling the amplified light in a single-mode fiber for optical control of the spatial mode, only about half of this power remains available for spectroscopy.

This source covers a spectral region of about $150 \mathrm{~nm}$ around $2 \mu \mathrm{m}$, and can be used for DCS of atomic and/or molecular transitions resonant in such spectral range. Among them, different overtones of fundamental vibrations of $\mathrm{CO}_{2}$ fall in this region $\left(\left(\mathrm{v}_{1} \mathrm{v}_{2}{ }^{l} \mathrm{v}_{3} \mathrm{r}\right)\right.$ HITRAN database notation [29] was used to denote vibrational modes). In particular, we investigated the $\left(20^{0} 11-00^{0} 01\right)$ rovibrational band of ${ }^{12} \mathrm{C}^{16} \mathrm{O}_{2}$ between 5064 and $5126 \mathrm{~cm}^{-1}$ to demonstrate the performance of our experimental approach.

A medium finesse FP is used both as a molecular absorption cell and as a spectral filter to resolve, in transmission, the coupled comb modes. It is hemifocal [with a dielectric plane mirror $(R>99.9 \%)$ and a 50-mm-focal-length metallic curved mirror $(R \approx 98 \%)]$ with a length $L \sim 3.3 \mathrm{~cm}$, and hence FSR in vacuum $\Delta_{\mathrm{FSR}}=c / 2 L \sim 4.5 \mathrm{GHz}$. The mirrors are mounted on kinematic mountings separated by three invar bars to get a highly passive length stability. The filtering ratio $m=\Delta_{\mathrm{FSR}} / f_{r}=18$ is kept constant by actively controlling $L$ with three stepped motors and a piezoceramic actuator mounted on the plane mirror support. A narrow modulation of the FP length $L$ and lock-in detection of a fraction of the FP transmitted light was used to keep all resonant FCL modes at the maximum transmission. The FP finesse of $F_{\text {vac }}=105$ under vacuum was determined by measuring the side mode suppression parameter, $\rho=\mathcal{T}\left(f_{r}, \Delta_{\mathrm{FSR}}, F\right)=-21 \mathrm{~dB}$ (i.e., the FP transmission at a frequency $f_{r}$ apart with respect to the nearest FP resonance). This FP configuration is the best compromise to get an efficient filtering at high Vernier ratio with an almost flat dispersive response throughout the whole FCL bandwidth. In addition, the absorption path length is increased up to $4 \mathrm{~m}$, which allows one to perform spectroscopic measurements within a wide dynamic range of sample pressures $\left(\sim 2\right.$ orders of magnitude for the $\mathrm{CO}_{2}$ transitions at $1.95 \mu \mathrm{m})$. To this aim, the FP is mounted inside a

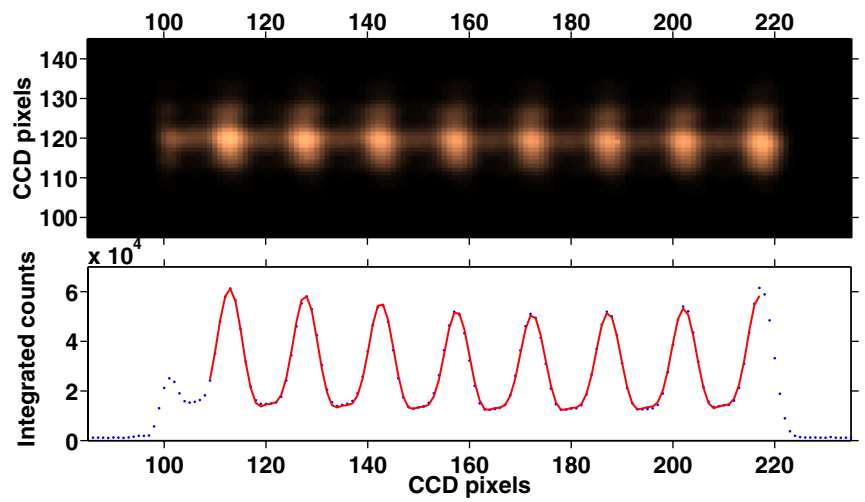

FIG. 2. (Color online) Top: Image of the eight resolved modes of the filtered comb around $2 \mu \mathrm{m}$. Bottom: Intensity of the comb modes of the above image, integrated in the vertical CCD direction (blue dots) and fit to a FP-SOPRA instrumental function (red line) (see text for details). As shown, the optical configuration of the commercial camera reduces the field of view in direct CCD illumination to 150 pixels instead of the nominal 320 ones.

vacuum chamber connected to a vacuum pump and to a system to fill the chamber with $\mathrm{CO}_{2}$ gas at a controlled pressure.

The FP transmission is $0.05 \%$, as expected from design, taking into account reflectivity and absorption of the FP mirrors, as well as the $m$ value. We use a 2-m-focal-length Fastie-Ebert monochromator (SOPRA) [27] to spectrally resolve this FP transmitted light. The echelle grating $(720 \mathrm{~g} /$ $\mathrm{mm}$ ) works at the blaze angle at the first order for $\lambda=1.9 \mu \mathrm{m}$, with a free spectral range of $\sim 5300 \mathrm{~cm}^{-1}$. A proper optical setup was chosen to match the FP transmitted light to the SOPRA F number (20:1). In these conditions, the SOPRA works at the diffraction limit configuration, corresponding to a final resolution (FWHM) of about $1.5 \mathrm{GHz}$ around $2 \mu \mathrm{m}$. Transmissive optical components were avoided to minimize dispersive as well as fringing effects, and to get an apparatus operating at any frequency in the whole FCL spectrum around $2 \mu \mathrm{m}$. The selected dispersed portion of the input filtered comb is then sent to the output slit by means of an automatized system that changes the grating angle. The output light, conveniently magnified with a reflective telescope, is sent to an InSb 320 x 256 CCD camera (FLIR SC7000).

In Fig. 2, we show an image of eight filtered comb modes as recorded by the CCD. Taking into account $\Delta_{\mathrm{FSR}}$, it corresponds to an interval of approximately $32 \mathrm{GHz}(\approx 1 \mathrm{~cm}$ ${ }^{-1}$ ) of the comb spectrum. We work with a magnification factor of 4 to integrate well the comb intensity contained in the instrumental detection function. Using a lower magnification, a larger comb spectrum could be detected but at the expense of the reduced integrated intensity dynamic range per comb mode. The angular grating scan allows the acquisition of consecutive, $1 \mathrm{~cm}^{-1}$ wide, filtered comb portions. In addition, this configuration allows normalization of the comb intensity variations in the neighborhood of the absorbing mode. The intensity content of each detected mode is measured by fitting the integrated image in the vertical direction (bottom graph of Fig. 2), to a function which takes into account the instrumental response of the combined FPSOPRA system. The image of a single-frequency cw diode laser at $2013 \mathrm{~nm}$, coupled to 
the FP-SOPRA system, is used to measure such instrumental function. This function is then replicated up to eight times with a fixed separation (i.e., $\Delta_{\mathrm{FSR}}$ in pixels). This separation, the intensities $I_{i}(i=1,8)$ of these eight replicas, and the position of one of them $p_{0}$ are varied to fit the comb image, as shown in the bottom panel of Fig. 2 (red line). The $I_{i}$ 's are the comb mode intensity used to recover the spectrum. The integration time of about $1 \mathrm{~ms}$ per image filters out rapidintensity fluctuations of the detected modes, with only longperiod fluctuations remaining. We have checked that such fluctuations have a linear behavior for time periods of few minutes, independently of the portion of filtered comb detected, the illuminated CCD area, and the comb frequency.

Spectroscopy is performed by scanning $f_{r}$. For each $\mathrm{CO}_{2}$ spectrum, the $f_{r}$ is changed by $\sim 2.5 \mathrm{kHz}$, corresponding to a frequency scan of about $\pm 700 \mathrm{MHz}$ in 40 steps of $\sim 35 \mathrm{MHz}$ each. For the detected mode which is absorbed by the molecule (namely, $i=4$ ), the absolute frequency $\nu^{\text {comb }}{ }^{4}$ for the $f_{r}$ value which establishes the maximum absorption is calculated by

$$
v_{4}^{\mathrm{comb}}=N_{4} f_{r} \pm f_{\mathrm{CEO}},
$$

with $f_{\text {CEO }}=20 \mathrm{MHz}$ and $N_{4}$ the comb order number to be determined by successive steps. First, the targeted $\mathrm{CO}_{2}$ transition is addressed though the SOPRA wavelength scale; second, it is addressed by determining unambiguously $N_{4}$ and the sign of $f_{\text {CEO }}$ by comparison of $v_{4}^{\text {comb }}$ with the HITRAN database [29] value for this transition, $v_{o}^{\text {Hitran }}$. The $N_{i}$ orders for the other detected modes are then automatically calculated by using the Vernier ratio order, $m=18$. Finally, the frequency scale of the spectrum is calculated by using Eq. (1) for each scanned $f_{r}$ value.

For each scanned frequency, the corresponding filtered comb image is recorded and the intensity of each detected mode is recovered. The intensity normalization of the molecular absorbed mode is made by using the average intensity of the other nonabsorbing modes, with the exclusion of the two closest, to avoid possible residual interference effects. All other possible combinations of the nonabsorbing comb modes to obtain an intensity reference for normalization, produce the same spectroscopic results at the present level of precision. We note that although long-term intensity fluctuations still remain after normalization, being independent of intensity normalization, we take them into account by adding a linear background in the fit procedure. The scans are performed with an automatized procedure, and the result is shown in the video clip (see Supplemental Material [30]). The $f_{r}$ value is changed in the forward and backward directions to minimize systematic effects due to the scan procedure. Each spectrum requires approximately $2 \mathrm{~min}$ acquisition time but an improvement of a factor of 2 could be easily achievable by optimizing dead time between image acquisitions due to the frequency change procedure, which was prudentially overset.

\section{RESULTS AND DISCUSSION}

Frequencies and other spectral parameters of the recorded transitions are determined by fitting the experimental spectrum to the FP transmission Airy function of the absorbed mode at the molecular frequency $v_{o}[31]$,

$$
\mathcal{T}(v)=\frac{T^{2} e^{-\alpha(v) L}}{\left(1-R e^{-\alpha(v) L}\right)^{2}+4 R e^{-\alpha(v) L} \sin ^{2}[\epsilon(\nu) / 2]},
$$

where $R$ is the FP reflectivity and $\alpha(\nu) L$ is the single-pass absorption. The FP transmission $T$ also includes the mirror absorption $A(T=1-R-A)$. The phase shift per round trip, $\epsilon(v)$, depends on the FP detuning through two different contributions: one $\left(\epsilon_{\mathrm{vac}}\right)$ is due to cavity-length variations of the empty FP and the other $\left(\epsilon_{\mathrm{dis}}\right)$ is due to refractive-index variation $\Delta n$ related to gas absorption [32],

$$
\epsilon(\nu)=2 \pi\left(\frac{\Delta-\Delta_{\mathrm{FP}}}{\Delta_{\mathrm{FSR}}}+\frac{\nu \Delta n}{\Delta_{\mathrm{FSR}}}\right)=\epsilon_{\mathrm{vac}}+\epsilon_{\mathrm{dis}} .
$$

Here, the vacuum FP detuning is expressed in terms of molecular resonance detuning $\Delta=v-v_{o}$ and of the FP mode detuning $\Delta_{\mathrm{FP}}=v_{\mathrm{FP}}-v_{o}$, which is close to zero, due to the FCL-FP lock. Nevertheless, we left it as a free parameter in the fit to take into account that the FP could not be locked exactly at the maximum of the FP order resonant with the FCL absorbing mode.

In the following, we consider a resonant transition described by a homogeneous process with a maximum absorption coefficient $\alpha_{o}^{h}$ and linewidth (FWHM) $\Gamma_{0}$, which is inhomogeneously broadened by the Doppler effect, with a Gaussian distribution characterized by a standard deviation $\sigma_{T}=v_{0} \sqrt{k T / M c^{2}}$. This is the case of the $\mathrm{CO}_{2}$ measurements shown where the homogeneous contribution is dominated by collisional processes due to gas pressure. In these conditions, the gas absorbance is given by a Voigt profile,

$$
\alpha(v) L=\frac{1}{\sqrt{2 \pi} \sigma_{T}} \int \frac{2 \alpha_{0}^{h} L}{1+4\left(\frac{\Delta \nu-f}{\Gamma_{o}}\right)^{2}} e^{-\frac{f^{2}}{2 \sigma_{T}^{2}}} d f
$$

with $f$ being the Doppler shift of each molecular velocity class. Considering the complex refractive index of the absorbing media $n=1+\Delta n+i \alpha /(2 k)$, with $k$ the absorbed mode wave vector, and taking into account the Kramers-Kroning relations, we can express $\epsilon_{\text {dis }}$ in terms of molecular absorption parameters as

$$
\epsilon_{\mathrm{dis}}(\nu)=\frac{1}{\sqrt{2 \pi} \sigma_{T}} \int \frac{2 \alpha_{0}^{h} L}{1+4\left(\frac{\Delta \nu-f}{\Gamma_{o}}\right)^{2}} \frac{\Delta v-f}{\Gamma_{o}} e^{-\frac{f^{2}}{2 \sigma_{T}^{2}}} d f
$$

As in [4], Eqs. (2)-(5) provide both amplitude and phase information about the sample, by measuring the area under the Airy transmission. In our case, owing to the system which is able to better resolve the contribution of each diffracted comb mode per detection unit of the array, we have a wide dynamic range to measure intensity variations of such modes. As a consequence, our method is well suited to measure with high precision small modifications of the absorption line profile, and hence to accurately evaluate its spectroscopic information. Moreover, with respect to other dispersive-DCS approaches, detecting the transmitted photons per mode on a large detection area can improve the ultimate detection limit, given by CCD 


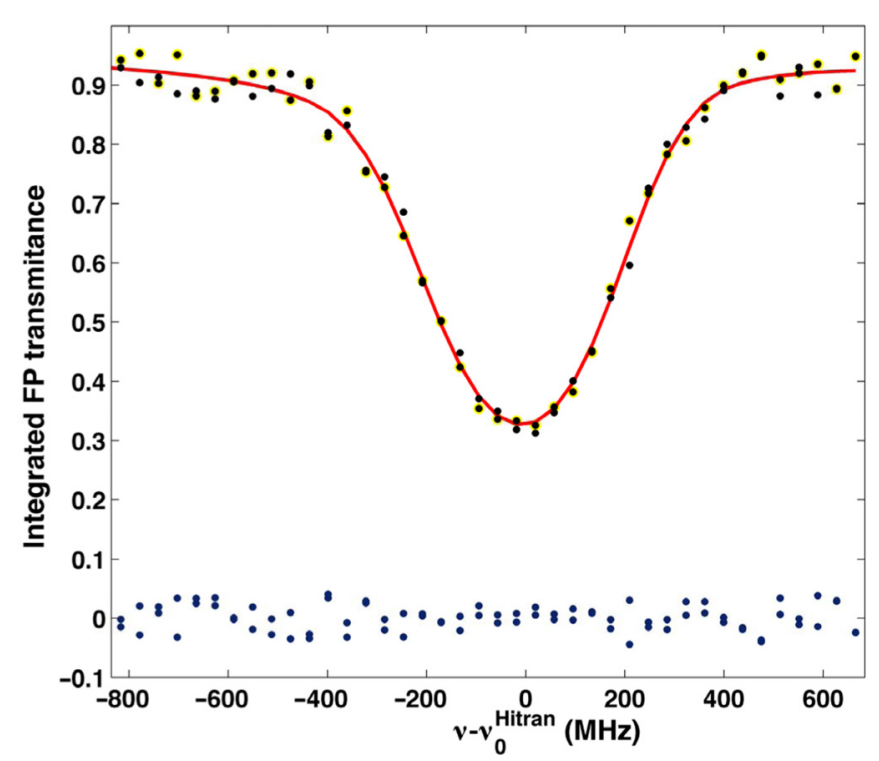

FIG. 3. (Color online) FP filtered FCL spectrum (black dots) of the $P(34)-\left(20^{0} 11-00^{0} 01\right) \mathrm{CO}_{2}$ transition at $5070.14 \mathrm{~cm}^{-1}$ and $P=10$ mbar, and fit (red line) to the function (2) plus linear background. Residuals (blue dots) are also shown. The values of the fixed parameters are $\sigma_{T}=282.359 \mathrm{MHz}, \mathrm{FSR}=4.5 \mathrm{GHz}, F_{\mathrm{vac}}=105$, and $m=18$. The fitted values of the line center $v_{o}=151999035.3(1.6)$ $\mathrm{MHz}$, line strength $S=1.32(5) \times 10^{-22} \mathrm{~cm} / \mathrm{mol}$, and collisional linewidth $\Gamma_{o}^{\mathrm{FWHM}}=52.0(9) \mathrm{MHz}$ agree with the values reported in the HITRAN database [29] for this transition, i.e., $v^{\text {Hitran }}{ }_{o}=$ $151999038.7(3.0) \mathrm{MHz}, S^{\text {Hitran }}=1.40(3) \times 10^{-22} \mathrm{~cm} / \mathrm{mol}$, and $\Gamma_{o}^{\text {Hitran }}=52.2(2.6) \mathrm{MHz}$. (See Supplemental Material for video movie of the spectrum scan.)

counting Poisson statistics. As an example, the strong thermal background present in the IR spectral region reduces the CCD counting range, which can be partially compensated by integrating the signal in a large detector area.

In the fit procedure, we have parametrized (2) in terms of transition $\left(\alpha_{o}^{h}, v_{o}, \Gamma_{o}\right.$, and $\left.\sigma_{T}\right)$ and $\mathrm{FP}\left(\Delta_{\mathrm{FSR}}, F_{\mathrm{vac}}, m\right.$, and $\left.\Delta_{\mathrm{FP}}\right)$ parameters to extract the relevant spectroscopic information. In addition, a linear background was added to take into account the nonperfect intensity normalization of the spectra. The $\alpha_{o}^{h}$, $v_{o}, \Gamma_{o}$, and $\Delta_{\mathrm{FP}}$ as well as linear background parameters are free in the fit, whereas $\sigma_{T}$, FSR, $F_{\text {vac }}$, and $m$ are fixed. In Fig. 3 , a fit to the spectrum of the $P(34)-\left(20^{0} 11-00^{0} 01\right) \mathrm{CO}_{2}$ transition at $5070.14 \mathrm{~cm}^{-1}$ is shown. The good agreement between experimental data and fit, as shown by the residuals, is a first indication of the validity of our model. The fitted $v_{o}, \Gamma_{o}$, and $\alpha_{o}^{h}$ parameters also agree with previously published values [29], taking into account the combined uncertainties. For the measured values, these uncertainties are mainly statistical deviations from the fit, limited by the signal-to-noise ratio of the spectrum. Systematic uncertainties due to possible $\sigma_{T}$ variations in the range of temperature stability of the gas $( \pm 1 \mathrm{~K})$ are added in quadrature to this uncertainty.

Figure 4 shows the powerfulness of our method to measure systematic effects related to tiny modifications of the absorption profiles. In the top panel, the behavior of $\Gamma_{o}$ and $v_{o}$ vs gas pressure for the $P(18)-\left(20^{0} 11-00^{0} 01\right) \mathrm{CO}_{2}$ transition at $5084.79 \mathrm{~cm}^{-1}$ is presented. The measured FWHM pressure self-broadening coefficient of $0.200(1) \mathrm{cm}^{-1} / \mathrm{atm}$ agrees with that tabulated for this transition [29] [i.e., $\left.0.201(10) \mathrm{cm}^{-1} / \mathrm{atm}\right]$, with a precision improvement of one order of magnitude. Moreover, we measured a self-collisional frequency shift coefficient of $0.010(6) \mathrm{cm}^{-1} / \mathrm{atm}$ for this transition by fitting the $v_{o}(P)$ values to a linear function. The intercept value of this fit (i.e., $v_{0}$ value at $P=0$ mbar) gives the line center frequency corrected by this self-collisional shift.

In order to thoroughly investigate systematic effects with our setup, we also measured possible light shifts (ac Stark shift). Therefore, measurements at different output power levels of the Tm-Ho FA were performed. The expected linear behavior of $\Gamma_{o}$ and $v_{o}$ vs the FCL mean power at the FA output is shown in the bottom panels of Fig. 4. Neither light broadening nor light shift above the quoted uncertainties was found. We only note an increment of the error bar with power, probably due to the increment of the mode-to-mode intensity fluctuations of the amplified FCL.

Finally, we evaluated the possible systematic effects due to the remaining comb light, not resonant with the molecular transition. To this aim, the DCS measurements were compared with those made with a FCL-assisted cw singlemode laser by using the same experimental setup. The comparison was performed for the $P(12)-\left(20^{0} 12-00^{\circ} 01\right) \mathrm{CO}_{2}$ transition at $4967.98 \mathrm{~cm}^{-1}$, where both the amplified FCL and $\mathrm{cw}$ laser operate. These rovibrational transitions belong to a different $\mathrm{CO}_{2}$ overtone band, about $122 \mathrm{~cm}^{-1}$ far from the other one shown in this work, confirming the broad spectral operation range of our DCS system. The results obtained, at a pressure of 1.3 mbar, yield $\nu_{o}^{\mathrm{OFC}}=148936405.8(1.0) \mathrm{MHz}$ and $v_{o}^{\mathrm{CW}}=148936405.4(9) \mathrm{MHz}$ for the line center, and $\Gamma_{0}^{\mathrm{OFC}}=8.34(9) \mathrm{MHz}$ and $\Gamma_{0}^{\mathrm{CW}}=8.34(9) \mathrm{MHz}$ for the homogeneous linewidth. Moreover, these values agree well with those tabulated for this transition at the same temperature and pressure conditions of the $\mathrm{CO}_{2}$ sample [29]. Therefore, there is no need to introduce a correction due to other noninteracting modes.

Taking into account the above analysis, we can infer a center frequency for the $P(18)-\left(20^{0} 11-00^{0} 01\right) \mathrm{CO}_{2}$ transition of 152438 073.0(9) MHz. The overall uncertainty comes from the sum in quadrature of those of the extrapolated frequency at zero pressure and of a possible light shift, both calculated from the fits of Fig. 4, and the accuracy of the comb frequency (about $300 \mathrm{~Hz}$ at these frequencies). This uncertainty is $\sim 300$ times smaller than the Doppler linewidth at room temperature, mainly limited by the signal-to-noise ratio of the spectra. An accuracy improvement of three times is achieved with respect to the previous reported value [29]. The relative precision of $6 \times 10^{-9}$ is comparable with frequency measurements of molecular transitions performed with dual-comb DCS in this spectral region [21]. In addition, an accurate determination of systematic errors as pressure and light shifts was performed with a DCS technique. Moreover, accuracies beyond ppb could be achieved with this technique, provided that an increased signal-to-noise ratio is achieved in narrower spectral shapes. In this case, however, particular attention should be paid to control or to avoid possible technique-dependent systematic effects, which may become relevant at this uncertainty level. 

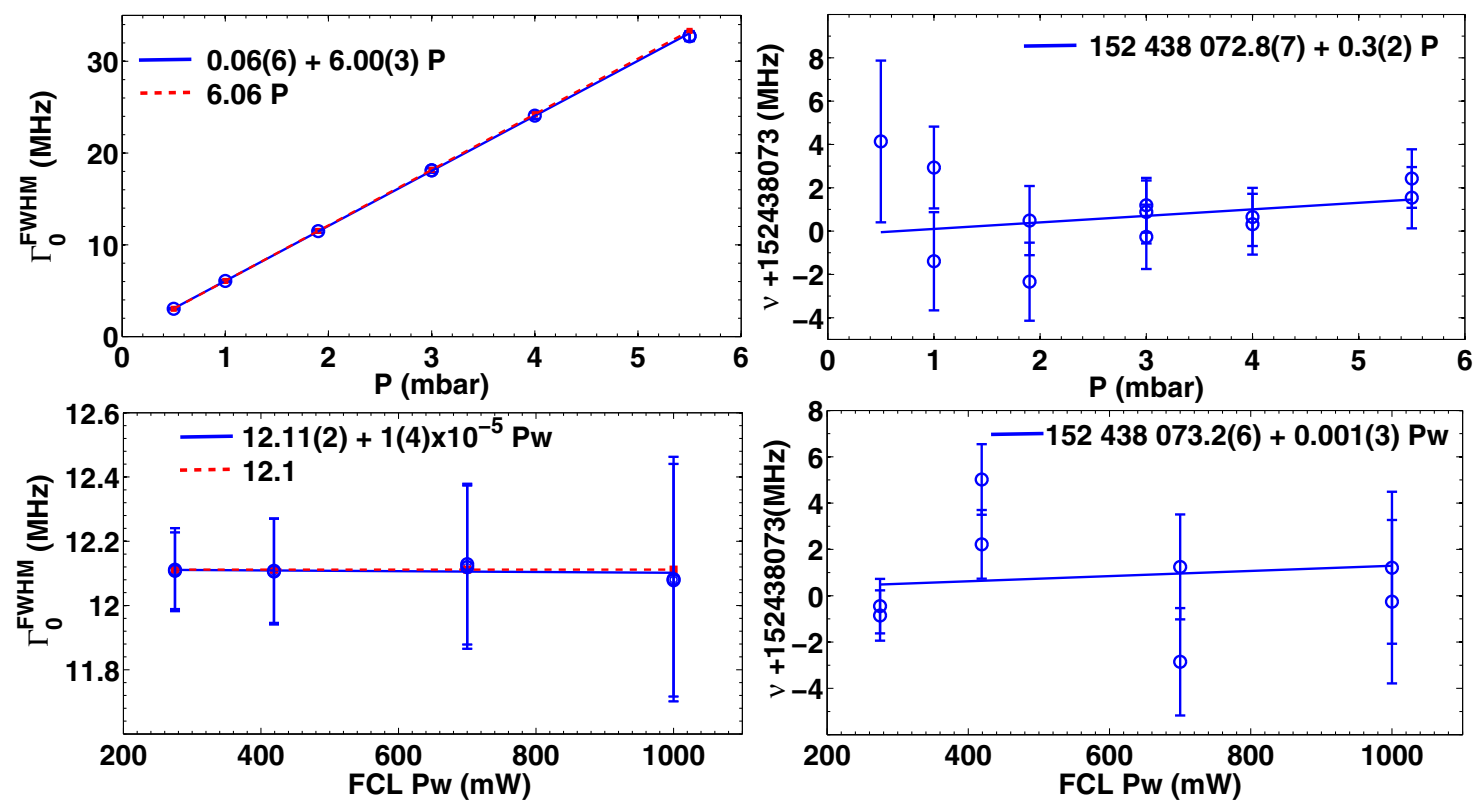

FIG. 4. (Color online) Behavior of the fitted spectroscopic parameters for the $P(18)-\left(20^{0} 11-00^{0} 01\right) \mathrm{CO}_{2}$ transition at $5084.79 \mathrm{~cm}^{-1}$ as a function of pressure and amplified FCL power. Top left: Homogeneous linewidth vs $\mathrm{CO}_{2}$ pressure (blue dots) and linear fit (blue line). Top right: Line center frequency vs pressure (blue dots) and linear fit (blue line). Bottom left: Homogeneous linewidth vs mean FCL power (blue dots) and linear fit (blue line). Bottom right: Line center frequency vs mean FCL power (blue dots) and linear fit (blue line). On the left panels, the Hitran data (red dots) [29] and line fit (red dashed line) are also shown for comparison.

In summary, we performed precise absolute frequency measurements of molecular transitions by using a Vernierdispersive DCS approach. The combined broad spectral coverage, accuracy in the frequency scale, and precise measurement of intensity line profiles should pave new ways to estimate the elusive effects in atomic and molecular systems.

[1] P. Maddaloni, P. Cancio, and P. De Natale, Meas. Sci. Technol. 20, 052001 (2009).

[2] A. Cingöz, D. C. Yost, T. K. Allison, A. Ruehl, M. E. Fermann, I. Hartl, and J. Ye, Nature (London) 482, 68 (2012).

[3] M. J. Thorpe, K. D. Moll, R. J. Jones, B. Safdi, and J. Ye, Science 311, 1595 (2006).

[4] C. Gohle, B. Stein, A. Schliesser, T. Udem, and T. W. Hänsch, Phys. Rev. Lett. 99, 263902 (2007).

[5] S. A. Diddams, L. Hollberg, and V. Mbele, Nature (London) 445, 627 (2007).

[6] I. Coddington, W. C. Swann, and N. R. Newbury, Phys. Rev. Lett. 100, 013902 (2008).

[7] B. Bernhardt, A. Ozawa, P. Jacquet, M. Jacquey, Y. Kobayashi, T. Udem, R. Holzwarth, G. Guelachvili, T. Hänsch, and N. Picqué, Nat. Photon. 4, 55 (2010).

[8] F. Keilmann, C. Gohle, and R. Holzwarth, Opt. Lett. 29, 1542 (2004).

[9] A. Schliesser, M. Brehm, F. Keilmann, and D. W. van der Weide, Opt. Express 13, 9029 (2005).

[10] Z. Zhang, T. Gardiner, and D. T. Reid, Opt. Lett. 38, 3148 (2013).

\section{ACKNOWLEDGMENTS}

This work was supported by the Italian Ministry of University and Research, Extreme Light Infrastructure-Italy (ELIItaly) project, and Progetti di Ricerca di Interesse Nazionale (PRIN), Project No. 2008H9F3ZY.

[11] T. Yasui, Y. Kabetani, E. Saneyoshi, S. Yokoyama, and T. Araki, Appl. Phys. Lett. 88, 241104 (2006).

[12] R. Grilli, G. Méjean, S. Kassi, I. Ventrillard, C. Abd-Alrahman, and D. Romanini, Environ. Sci. Technol. 46, 10704 (2012).

[13] S. Boudreau, S. Levasseur, C. Perilla, S. Roy, and J. Genest, Opt. Express 21, 7411 (2013).

[14] M. Brehm, A. Schliesser, and F. Keilmann, Opt. Express 14, 11222 (2006).

[15] T. Ideguchi, S. Holzner, B. Bernhardt, G. Guelachvili, T. Hänsch, and N. Picqué, Nature (London) 502, 355 (2013).

[16] I. Coddington, W. C. Swann, and N. R. Newbury, Phys. Rev. A 82, 043817 (2010).

[17] A. Foltynowicz, T. Ban, P. Maslowski, F. Adler, and J. Ye, Phys. Rev. Lett. 107, 233002 (2011).

[18] F. Zhu, T. Mohamed, J. Strohaber, A. A. Kolomenskii, T. Udem, and H. A. Schuessler, Appl. Phys. Lett. 102, 121116 (2013).

[19] T. Ideguchi, A. Poisson, G. Guelachvili, N. Picqué, and T. Hänsch, Nat. Commun. 5, 3375 (2014).

[20] F. Adler, M. J. Thorpe, K. C. Cossel, and J. Ye, Annu. Rev. Anal. Chem. 3, 175 (2010). 
[21] A. Zolot, F. Giorgetta, E. Baumann, W. Swann, I. Coddington, and N. Newbury, J. Quantum Spectrosc. Radiat. Transfer 118, 26 (2013).

[22] E. Baumann, F. R. Giorgetta, W. C. Swann, A. M. Zolot, I. Coddington, and N. R. Newbury, Phys. Rev. A 84, 062513 (2011).

[23] L. Moretti, A. Castrillo, E. Fasci, M. D. De Vizia, G. Casa, G. Galzerano, A. Merlone, P. Laporta, and L. Gianfrani, Phys. Rev. Lett. 111, 060803 (2013).

[24] C. Lemarchand, S. Mejri, P. L. T. Sow, M. Triki, S. K. Tokunaga, S. Briaudeau, C. Chardonnet, B. Darquie, and C. Daussy, Metrologia 50, 623 (2013).

[25] J. J. Hudson, B. E. Sauer, M. R. Tarbutt, and E. A. Hinds, Phys. Rev. Lett. 89, 023003 (2002).

[26] E. Reinhold, R. Buning, U. Hollenstein, A. Ivanchik, P. Petitjean, and W. Ubachs, Phys. Rev. Lett. 96, 151101 (2006).
[27] V. Mazzacurati, P. Benassi, and G. Ruocco, J. Phys. E 21, 798 (1988).

[28] N. Coluccelli, M. Cassinerio, P. Laporta, and G. Galzerano, Opt. Lett. 38, 2757 (2013).

[29] L. Rothman et al., J. Quant. Spectrosc. Radiat. Transfer 130, 4 (2013).

[30] See Supplemental Material at http://link.aps.org/supplemental/ 10.1103/PhysRevA.91.012505 for video clip showing the scan and detection procedure of the spectrum of the $P(34)$ $\left(20^{0} 11-00^{0} 01\right) \mathrm{CO}_{2}$ transition at $10 \mathrm{mbar}$.

[31] Y. Zhu, D. J. Gauthier, S. E. Morin, Q. Wu, H. J. Carmichael, and T. W. Mossberg, Phys. Rev. Lett. 64, 2499 (1990).

[32] In this analysis, we have considered the dispersive effects due to mirror coating independent of the frequency in the spectrum scan range, and hence included in the FP-FSR. 